\title{
Apocynin alleviated hepatic oxidative burden and reduced liver injury in hypercholesterolaemia
}

\author{
Long-Sheng $\mathrm{Lu}^{1}$, Chau-Chung $\mathrm{Wu}^{2,3,4}$, Li-Man Hung ${ }^{5}$, Meng-Tsan Chiang ${ }^{6}$, Ching-Ting Lin ${ }^{7}$, Chii-Wann \\ $\mathrm{Lin}^{7}$ and Ming-Jai Su${ }^{1}$ \\ 1 Graduate Institute of Pharmacology, National Taiwan University College of Medicine, Taipei, Taiwan \\ 2 Departments of Primary Care Medicine, National Taiwan University College of Medicine, Taipei, Taiwan \\ 3 Departments of Internal Medicine, National Taiwan University College of Medicine, Taipei, Taiwan \\ 4 Department of Internal Medicine, Eda Hospital, Kaohsiung, Taiwan \\ 5 Department of Life Science, Chang-Gung University, Taoyuan, Taiwan \\ 6 Department of Food Science, National Taiwan Ocean University, Keelung, Taiwan \\ 7 Graduate Institute of Biomedical Engineering, National Taiwan University, Taipei, Taiwan
}

\section{Keywords}

apocynin - cholesterol - gp91 ${ }^{\text {phox }}$ - liver injury

$-\mathrm{NADPH}$ oxidase

\section{Correspondence}

Professor Ming-Jai Su, 11F No. $1 \mathrm{Sec} .1$ Ren-Ai Rd., Taipei 10051, Taiwan.

Tel: +886223123456 ext 8317

Fax: +886223971403

e-mail: mjsu@ha.mc.ntu.edu.tw

Received 16 August 2006

accepted 16 December 2006

DOI:10.1111/j.1478-3231.2007.01451.x

\begin{abstract}
Background: This study addressed the effects of apocynin, a nicotinamide adenine dinucleotide phosphate (NADPH) oxidase inhibitor, on hepatic oxidative burden and liver injury during diet-induced hypercholesterolaemia. Methods and Results: Male Wistar rats were fed a $4 \%$ cholesterol-enriched diet for 3 weeks. Apocynin was administered in drinking water concurrently. The high-cholesterol diet (HC) significantly increased the serum level of cholesterol and hepatic cholesterol ester deposition, and these parameters were similar between the HC and high-cholesterol diet plus apocynin (HCA) groups. The HC group showed abnormal liver function tests [alanine aminotransferase (ALT), aspartate aminotransferase (AST), and alkaline phosphatase (Alk-P)] as well as increased Evans blue extravasation and macrophages infiltration. Apocynin treatment could suppress these inflammation-related parameters. In vivo measurement of $\mathrm{NADPH}$-derived cellular autofluorescence suggested that HC increased oxidative stress in hepatocytes. Biochemical analysis of redox status including thiobarbituric acid reactive substances, reduced glutathione, and oxidized glutathione also confirmed the phenomenon. Apocynin treatment was able to alleviate these indices of oxidative burden owing to HC. Furthermore, apocynin-abrogated HC induced gp $91^{\text {phox }}$ expression, suggesting the involvement of NADPH oxidase in the pathogenesis. Conclusions: We concluded that apocynin suppressed NADPH oxidase activation and subsequent liver injuries owing to high-cholesterol intake in rats. The impacts of cholesterol metabolism disorders on pathogenesis and progression of steatohepatitis warrant further clinical investigation.
\end{abstract}

Non-alcoholic fatty liver diseases (NAFLD) have recently received considerable attention. As diabetes, dyslipidaemia and obesity may become pandemic disorders in the coming decades, NAFLD might emerge as a leading risk factor for cirrhosis and hepatocellular carcinoma $(1,2)$. While 'fatty liver' in most of the literature is assumed to be the result of triglyceride accumulation, the pathogenic potential of other lipids should also be considered. Cholesterol is a lipid of considerable biological importance, and during experimental hypercholesterolaemia, significant liver steatosis can be found. There are ample evidences that low-density lipoprotein (LDL) exposure and cholesterol overload could induce cellular and tissue damages (3-8). Although under normal conditions the liver is spared from cholesterol toxicity because of regulated intake and efficient esterification, it has been demonstrated that cholesterol overload results in liver inflammation and fibrosis $(9,10)$. However, the underlying mechanism remains to be explored.

One of the common cellular responses to cholesterol overload or hypercholesterolaemia is induction of free radical production and increased oxidative burden. Studies in atherosclerosis have demonstrated that an imbalanced redox status may induce inflammation and result in macrophage infiltration. Nicotinamide 
adenine dinucleotide phosphate oxidase (NADPH oxidase), a pentameric free radical-producing complex, is a major source of cellular reactive oxygen species (ROS). Increased NADPH oxidase activity has been implicated in macrophage activation and vascular inflammation (11). Expressions of NADPH oxidase subunits were confirmed in hepatocytes, Kupffer cells, and stellate cells. Among these subunits, gp91 ${ }^{\text {phox }}$ is the major catalytic subunit responsible for the ROS generation capability of the complex. It is possible that cholesterol overload might result in NADPH oxidase activation in the liver and trigger inflammatory injuries. Therefore, the specific purpose of this study was to test this hypothesis and to determine whether apocynin, an NADPH oxidase inhibitor, would affect oxidative stress and inflammatory injuries in the liver during hypercholesterolaemia.

\section{Methods}

\section{Animals and sample preparation}

Animal experiments have been conducted according to the institute regulations and approved by the ethic committee of National Taiwan University College of Medicine. Male Wistar rats (around $250 \mathrm{~g}$ ) were randomly assigned to a high-cholesterol diet (HC), a high-cholesterol diet plus apocynin (HCA), standard chow (C), and standard chow plus apocynin (A) groups for 3 weeks. A HC diet consists of $4 \%$ cholesterol in addition to the regular recipe of standard chow. Apocynin, a plant-derived NADPH oxidase inhibitor, was added into drinking water at a concentration of $1.5 \mathrm{mM}$ from the first day of the experiment. For liver sample preparation, rats received an overdose intraperitoneal (i.p.) injection of pentobarbital $(100 \mathrm{mg} / \mathrm{kg})$ before being killed. After exsanguination and retrograde perfusion through the abdominal aorta, the left lobe of the liver was quickly removed, cut into cubes (around $200 \mathrm{mg}$ ), snap-frozen in liquid nitrogen, and stored at $-80^{\circ} \mathrm{C}$ until processing. For blood sampling, blood samples were acquired with an intracardiac puncture after anaesthesia with pentobarbital $(65 \mathrm{mg} / \mathrm{kg}$ i.p.).

\section{Microvascular leakage assay}

Hepatic microvascular permeability was evaluated with the Evans blue leakage method, and six rats from each group were included in the experiment. A bolus of Evans blue $(30 \mathrm{mg} / \mathrm{kg})$ was injected via the jugular vein. After $30 \mathrm{~min}$, rats were exsanguinated and retrogradely perfused with phosphate-buffered saline (PBS) to wash out excess intravascular dye. Residual dye in a
$500 \mathrm{mg}$ block from the left lobe liver was extracted with formamide at $60^{\circ} \mathrm{C}$ for $72 \mathrm{~h}$. The extract was centrifuged and $300 \mu \mathrm{l}$ supernatant was measured with a spectrophotometer for absorbance at $620 \mathrm{~nm}$. The concentration of the dyes in the extract was calculated from a standard curve of Evans blue in formamide. The tissue concentration of the dyes was expressed as microgram dye per gram of tissue.

\section{Biochemistry}

The serum levels of total cholesterol, LDL, highdensity lipoprotein (HDL), triglyceride, alanine aminotransferase (ALT), aspartate aminotransferase (AST), and alkaline phosphatase (Alk-P) were determined with commercial colurimetric kits (Dade Behring, Marburg, Germany). Samples from eight rats in each group were included. To determine the liver lipid contents, fresh exsanguinated liver blocks (from four rats in each group) were homogenized and tissue lipids were extracted according to Carlson and Goldsford's methods with modifications (12). Lipid extracts were then emulsified, and commercial kits were applied to determine the concentration of total cholesterol (Audit Diagnostics, Cork, Ireland), free cholesterol (Wako Pure Chemical Industries, Osaka, Japan), and triglyceride (Randox Laboratories, Crumlin, UK). Data were expressed as $\mathrm{mg}$ lipid per gram of tissue.

\section{Biochemical analysis of redox status}

For thiobarbituric acid-reactive substance (TBARS) measurement, samples ( $n=6$ in each group) were homogenized in PBS. Hundred microliters of tissue homogenates were then mixed with an equal volume of $10 \%$ trichloroacetic acid for $15 \mathrm{~min}$ and centrifuged at $1500 \mathrm{~g}$ for $10 \mathrm{~min}$. Hundred microliters supernatant was mixed with an equal volume of $0.67 \%$ thiobarbituric acid incubated in a water bath at $95^{\circ} \mathrm{C}$. Then, the samples were centrifuged at $1500 \mathrm{~g}$ for $10 \mathrm{~min}$ and the TBARS concentration was spectrophotometrically measured at $530 \mathrm{~nm}$ against a saline blank. The TBARS was determined from the standard curve that was constructed with tetramethoxypropane and was expressed as $\mathrm{nmol} / \mathrm{mg}$ protein. The amount of protein was determined by the Bradford method.

Reduced glutathione (GSH) and oxidized glutathione (GSSG) levels were analyzed with a commercially available kit according to the manufacturer's instruction (Glutathione assay kit; Cayman Chemical, Michigan, MI, USA). Briefly, frozen samples ( $n=6$ in each group) were cut into cubes (around $200 \mu \mathrm{g}$ ) and homogenized in PBS on ice. After centrifugation at $2000 \mathrm{~g}$ for $2 \mathrm{~min}$, the supernatant was sequentially 
deproteinated with $10 \%$ metaphosphoric acid and $4 \mathrm{M}$ triethanolamine. The sample was then collected, freshly prepared assay buffer (provided by the manufacturer) was added, and incubated in the dark on an orbital shaker. Absorbance was read at $405 \mathrm{~nm}$ using a microtiter plate reader. A standard curve was generated using known concentrations of GSH and GSSG. Results were expressed in micromoles (for GSH) and nanomoles (for GSSG) per gram of tissue.

\section{Immunoblotting}

Samples were homogenized in protein extraction buffer (mammalian protein extraction reagent; Pierce Biotechnology, Rockford, IL, USA). Tissue lysates from four animals in each group were pooled for further analysis. Fifty-microgram proteins were mixed with electrophoresis buffer, boiled, and electrophoresized on $4-10 \%$ gradient acrylamide gels. After electrophoresis, proteins were transferred onto a polyvinylidene difluroide (PVDF) membrane and the membrane was blocked with 5\% non-fat milk for $1 \mathrm{~h}$. Then, it was incubated with a 1:100 diluted goat antigp91 ${ }^{\text {phox }}$ antibody (Santa Cruz Biotechnology, Santa Cruz, CA, USA) at $4{ }^{\circ} \mathrm{C}$ overnight. Unbound primary antibodies were washed out and the membrane was incubated with PBS-diluted horseradish peroxidaseconjugated secondary antibodies in room temperature for $60 \mathrm{~min}$. The horseradish peroxidase was then detected with enhanced chemiluminescence (Pierce Biotechnology) and documented on an autoradiographic film. The films were scanned with a densitometer and quantified with IMAGEJ.

\section{Immunohistochemistry}

A tissue block was processed to obtain cryosections of $5 \mu \mathrm{m}$ thickness. We used the DAB kit (EnVision; DAKO, Glostrup, Germany) to perform immunohistochemistry. Cryosections were fixed with cold methanol and incubated with monoclonal primary antibody against ED1 in 1:100 dilutions at room temperature for $60 \mathrm{~min}$. The sections were then incubated with a detection secondary antibody for $30 \mathrm{~min}$ and developed with a substrate-chromogen solution.

\section{Two-photon excitational microscopy}

Four rats in each group were included in this experiment. The two-photon microscopy system was moderated from an inverted microscope (Olympus IX71, Center Valley, PA, USA). The light source was a Ti-sapphire mode-lock pulse laser (Spectra-physics Tsunami, Spectra-Physics, Stratford, CT, USA) with a pump laser (533 nm, Spectra-Physics Millennia, Spectra-Physics). The incident laser for observation was set to be $780 \mathrm{~nm}$ and $\sim 5 \mathrm{~mW}$ on the specimen. The objective used in the experiments was $\times 20$ air objective, NA $=1.2$ (Olympus, UPlanApo, Center Valley, PA, USA), and NADPH fluorescence was collected with a band filter $(560-680 \mathrm{~nm})$. As NADPH is one of the major fluorescent cellular electron carriers, measurement of NADPH-derived autofluorescence might reflect oxidative phosphorylation and cellular metabolism (13-15). For intravital microscopy of the liver, a $3 \mathrm{~cm}$ midline incision allowed exposure of the freed left lobe of the liver in an anesthetized rat, and the position of the scanning area was adjusted with a translation stage. Data were processed into gray-scale micrographs and hepatocytes were identified on the basis of typical hexagonal morphology. The cellular autofluorescence intensity (AFI) of the hepatocytes was analyzed with ImageJ. Stellate cells and Kupffer cells were not included in the analysis as we were not able to identify these cells in the absence of adequate markers.

\section{Data analysis}

All data were expressed as mean \pm SEM. Data were analyzed with unpaired two-tailed Students' $t$-test, and significance was defined as $P<0.05$.

\section{Results}

Apocynin had no effects on serum lipid profile and liver lipid composition during high-cholesterol intake.

The serum lipid profile of rats on cholesterolenriched diet is shown in Fig. 1. Feeding rats on the $4 \%$ cholesterol-enriched diet led to significant changes in the serum cholesterol level, which was mainly because of increased LDL but not HDL. There was no significant difference in triglyceride. Apocynin treatment had no effects on the serum lipid profile either in control or cholesterol-fed rats. The lipid composition of the liver is shown in Fig. 2. High-cholesterol intake resulted in a 10-fold elevation in total cholesterol and a threefold increase in triglyceride in the liver. As the concentration of free cholesterol remained constant, the excess cholesterol was stored in ester form. Apocynin treatment did not alter the lipid composition of livers from either control or cholesterol-fed animals.

\section{Apocynin reversed high-cholesterol intake-induced hepatic inflammation}

Analysis of serum markers of liver functions (ALT, AST, and Alk-P) is shown in Fig. 3. A HC resulted in 

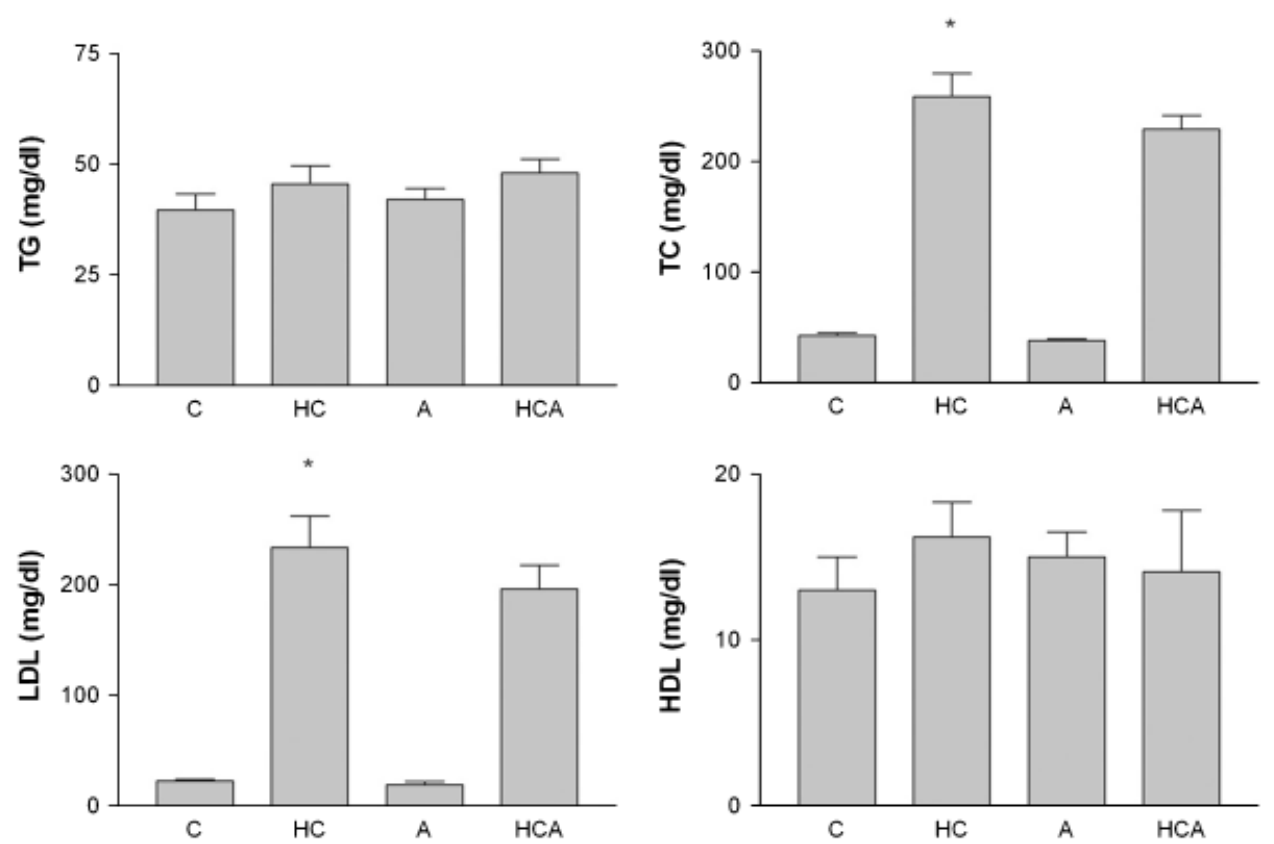

Fig. 1. Serum lipid profiles of control (C), apocynin (A), high-cholesterol intake (HC), and high-cholesterol intake treated with apocynin (HCA) groups. Data were from eight animals in each group. ${ }^{*} P<0.05$ compared with $C$.

moderately elevated ALT, AST, and Alk-P. Apocynin treatment had no effects on liver function tests in control rats but decreased HC-induced elevations in ALT and Alk-P, while reduction in AST did not reach statistical significance. As increased microvascular permeability is a feature of inflammation, we quantified Evans blue leakage in the liver, and the results are shown in Fig. 4. An increase in dye leakage was found in the livers from the HC group and apocynin treatment reversed this phenomenon. Immunohistochemical analysis of inflammation is shown in Fig. 5. In accordance with serum biochemical markers and dye leakage analysis, we found the presence of ED1positive macrophages in the livers from the HC group. Apocynin treatment alone did not influence the density of ED1-positive macrophages but it reduced the macrophage density in hypercholesterolaemic livers by $47 \%$.

\section{Apocynin alleviated hepatic oxidative burden during HC}

Measurement of NADPH-derived cellular autofluorescence with intravital two-photon excitational microscopy provides an imaging-based assay of oxidative metabolism in hepatocytes in vivo, as low autofluorescence intensity reflects decreased amount of cellular NADPH owing to enhanced NADPH oxidation (16). The typical figures and analysis of cellular AFI are shown in Fig. 6. We found a decreased AFI in hepatocytes from the HC group when compared with the $\mathrm{C}$ group. On the contrary, an increased AFI was found in the HCA group compared with the HC group. To further determine the hepatic oxidative burden, the liver contents of GSH, GSSG, and TBARS were measured, as shown in Fig. 7. We found decreased GSH, increased GSSG, and increased TBARS content in the livers from the HC group when compared with the $\mathrm{C}$ group. Apocynin treatment reversed depleted tissue glutathione in the HC group. It also decreased the TBARS content in the HC group but not in the control group. As apocynin is an NADPH oxidase inhibitor, we measured the expression of the major catalytic subunit $\mathrm{gp} 91^{\text {phox }}$ in the livers. As shown in Fig. 8, an increased expression of gp91 ${ }^{\text {phox }}$ was found in the HC group, and it was corrected with apocynin treatment.

\section{Discussions}

In this study, we demonstrated that apocynin reversed HC-induced liver injury, including abnormal liver function tests, microvascular leakage, and inflammatory infiltrates of macrophages. Its effects on reduced expression of gp91 ${ }^{\text {phox }}$, decreased TBARS, replenished cellular GSH, and NADPH all alleviated hepatic oxidative burden during hypercholesterolaemia and might underlie its therapeutic potency.

Apocynin is isolated from the immunomodulatory components of Picrorhiza kurroa extracts. It interferes 

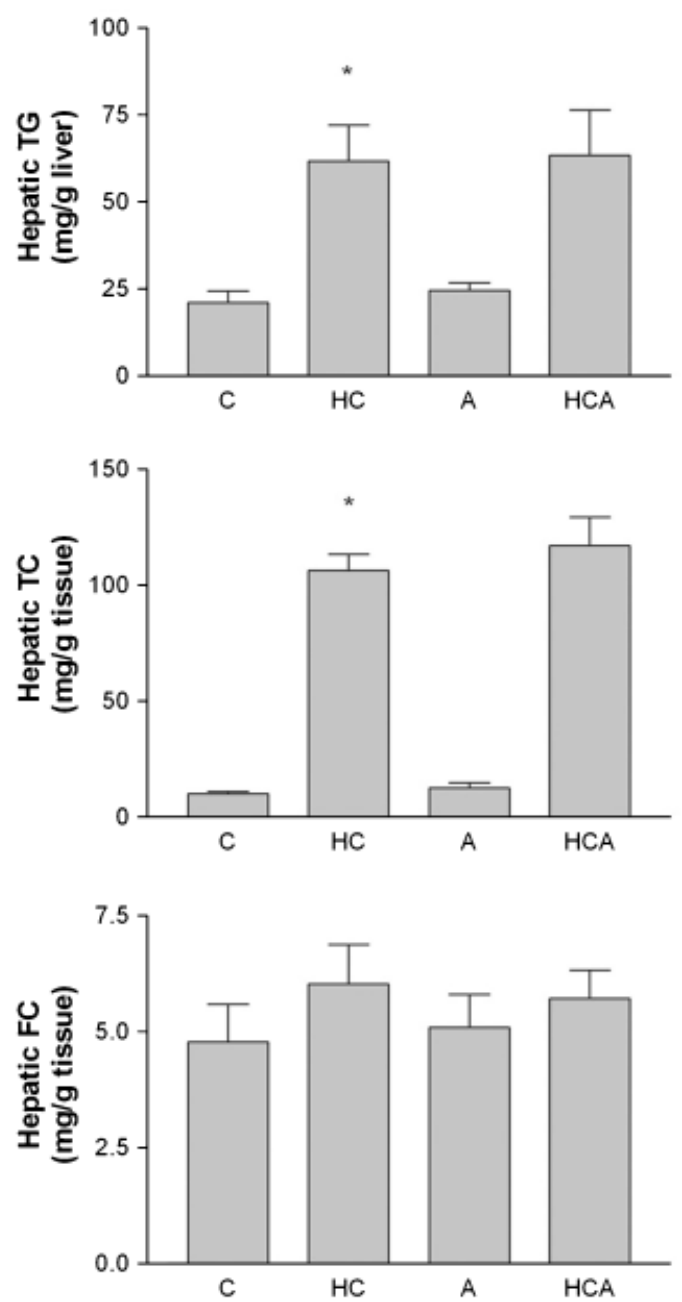

Fig. 2. Analysis of hepatic content of triglyceride (TG), total cholesterol (TC), and free cholesterol (FC). Data were from four animals in each group. ${ }^{*} P<0.05$ compared with control (C).

with membrane assembly of cytosolic subunits of $\mathrm{NADPH}$ oxidase and hence is a strong NADPH oxidase inhibitor. Oxidative stress derived from NADPH oxidase has been implicated in many liver diseases, including cirrhosis (17), ischaemia reperfusion injury (18), hepatocellular carcinoma (19), haemorrhagic shock (20), and acetaminophen-induced liver toxicity (21). Cells often respond to cholesterol loading with enhanced intracellular oxidative stress. The NADPH oxidase complex is an important source of intracellular ROS, and it is also an important mediator in ROS production related to cholesterol exposure $(22,23)$. Cholesterol might induce NADPH oxidase activation via activation of $\mathrm{PKC} \alpha$ (23), increased ER stress $(22,24)$, and assembly of subunits on the cholesterol-rich membrane microdomain (25). We found that administration of apocynin alleviated in-
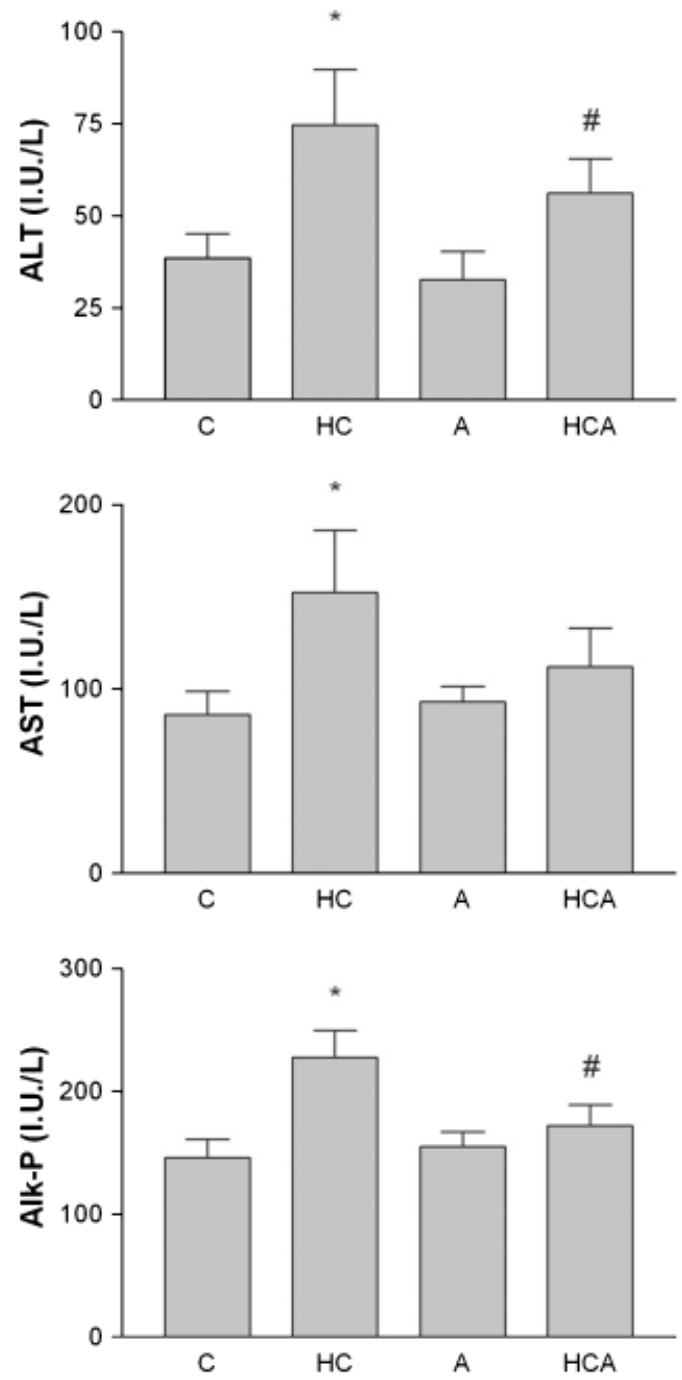

Fig. 3. Analysis of biochemical markers [alanine aminotransferase (ALT), aspartate aminotransferase (AST), and alkaline phosphatase (Alk-P)] of liver injury. Data were from eight animals in each group. ${ }^{*} P<0.05$ compared with control $(C)$; ${ }^{\#} P<0.05$ compared with high-cholesterol intake $(\mathrm{HC})$.

dices of hepatic oxidative stress in cholesterol-fed rats. It is interesting that apocynin also corrected gp $91^{\text {phox }}$ upregulation. Both evidences suggested that NADPH oxidase is involved in hepatic ROS production during hypercholesterolaemia. In the meantime, other sources of ROS might participate in cholesterol-induced liver injury. A recent report demonstrated that mitochondrial cholesterol overload resulted in mitochondrial glutathione depletion and sensitized steatotic livers to tumor necrosis factor-induced steatohepatitis (26). As apocynin inhibits NADPH oxidase via interrupted membrane assembly but not subunit expression, it is also possible that induction of gp91 ${ }^{\text {phox }}$ expression during hypercholesterolaemia is 


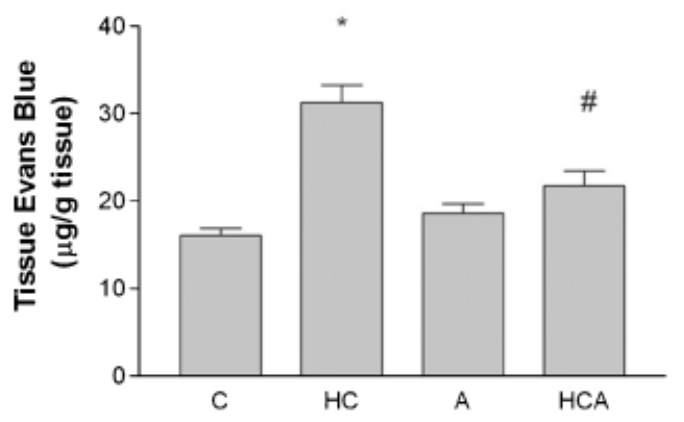

Fig. 4. Analysis of hepatic microvascular leakage. Data were from six animals in each group. ${ }^{*} P<0.05$ compared with control (C); ${ }^{*} P<0.05$ compared with high-cholesterol intake $(\mathrm{HC})$.
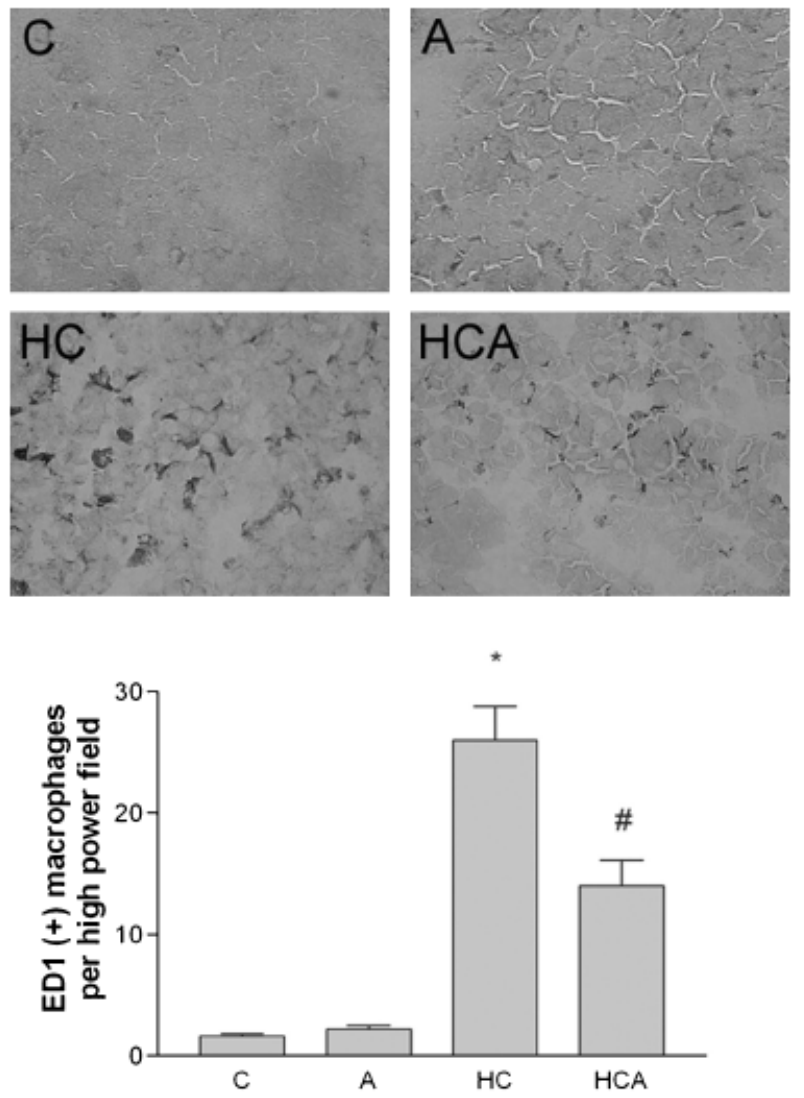

Fig. 5. Analysis of inflammatory infiltration of monocytes/ macrophages. The upper panel shows micrographs of immunohistochemical labeling of ED1-positive invading macrophages (under $\times 200$ magnification), and the lower panel shows a bar graph of the quantification results. For quantification, samples from three animals in each group were prepared for immunohistochemical labeling, and five highpower fields in each slide were counted. ${ }^{*} P<0.05$ compared with control (C); $P<0.05$ compared with high-cholesterol intake $(\mathrm{HC})$.
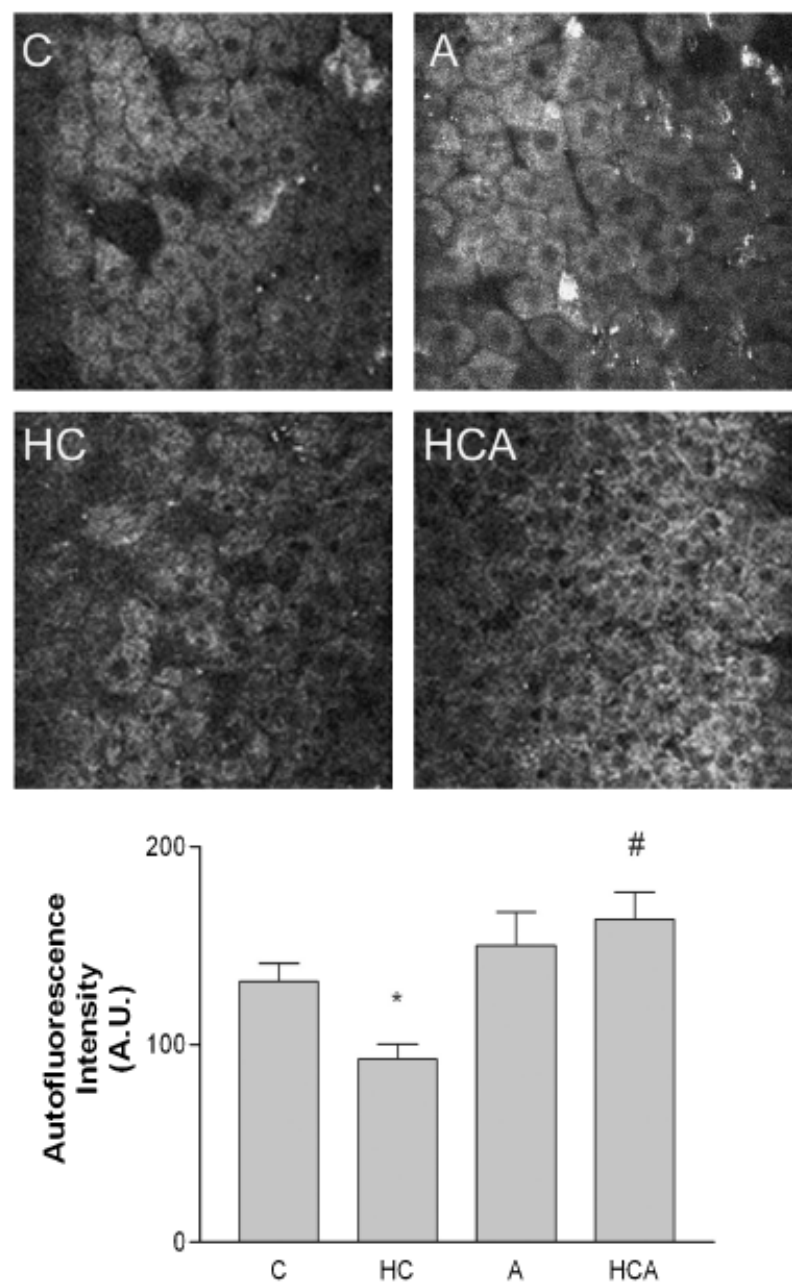

Fig. 6. Intravital two-photon excitational microscopy for in vivo imaging of oxidative burden in the liver. Representative images are shown in the upper panel (under $\times 200$ magnification). A bar graph of quantitative autofluorescence intensity analysis is shown in the lower panel. For quantitative analysis, five hepatocytes in each image were manually segmented and values of autofluorescence intensity were averaged to represent this image. Three images were taken from each animal and four animals in each group were analyzed. ${ }^{*} P<0.05$ compared with control (C); ${ }^{\#} P<0.05$ compared with high-cholesterol intake $(\mathrm{HC})$.

associated with the pro-oxidative environment and this would result in a vicious cycle for ROS production. Apocynin treatment possibly breaks this cycle from the very beginning, and this mechanism might underlie its therapeutic efficacy.

We found that feeding a very high percentage (4\%) of cholesterol led to 10 -fold cholesteryl ester accumulation, while there were no significant changes in the hepatic content of free cholesterol. The manipulation of intracellular cholesterol esterification has been reported to be associated with inflammatory 

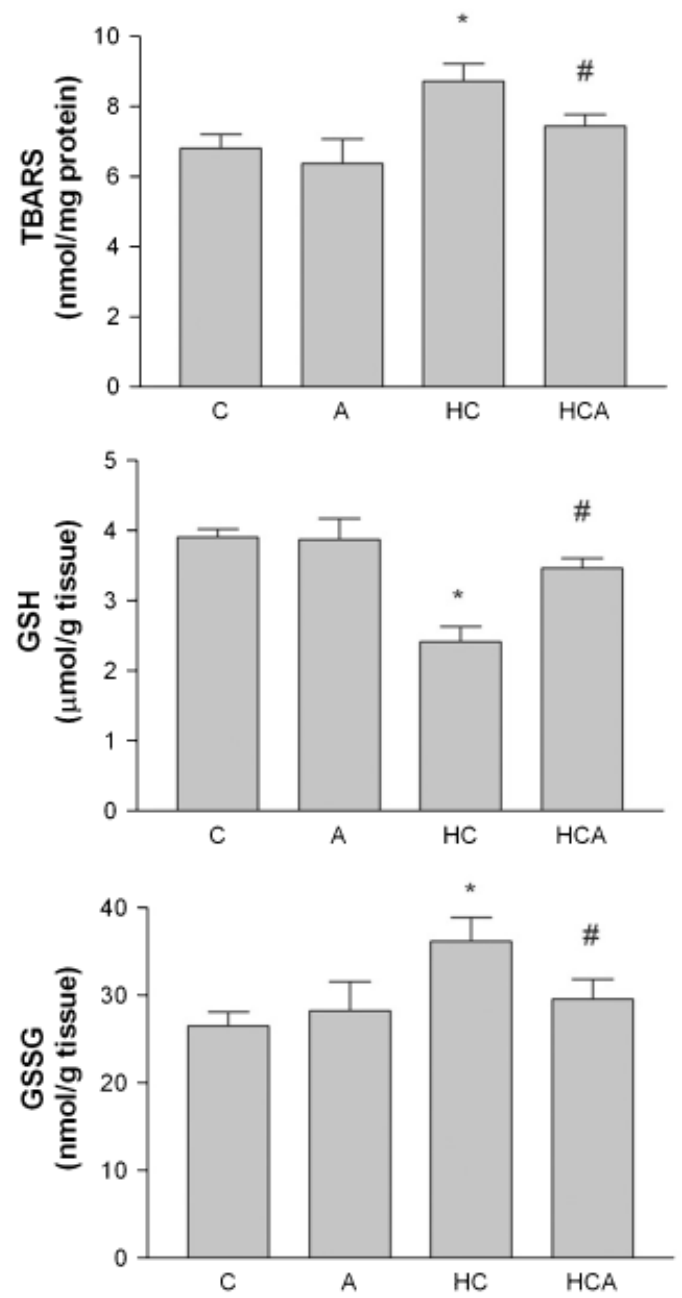

Fig. 7. Biochemical analysis of hepatic oxidative burden. Tissue contents of thiobarbituric acid reactive substances (TBARS), reduced glutathione (GSH), and oxidized glutathione (GSSG) were measured, and results are shown in bar graphs. Data were from six animals in each group. ${ }^{*} P<0.05$ compared with control (C); ${ }^{\#} P<0.05$ compared with high-cholesterol intake $(\mathrm{HC})$.

responses. ACAT2 deletion and subsequently decrease of intracellular cholesteryl ester, was protective against macrophage transformation to foamy cells, inflammatory gene expressions, and atherosclerosis (27). On the contrary, liposomal acid lipase deletion, which would cause cellular cholesteryl ester accumulation, resulted in neutrophilic infiltration, and inflammatory lung damage (28). In humans with liposomal acid lipase deficiency, hepatosplenomegaly, and liver cirrhosis have also been reported (29). Therefore, it is possible that cellular accumulation of cholesteryl ester might be an inflammation trigger in the liver of cholesterol-fed rats.

$\mathrm{NADPH}$ is a major fluorochrome in cells. Upon oxidation, it is converted to nicotinamide adenine
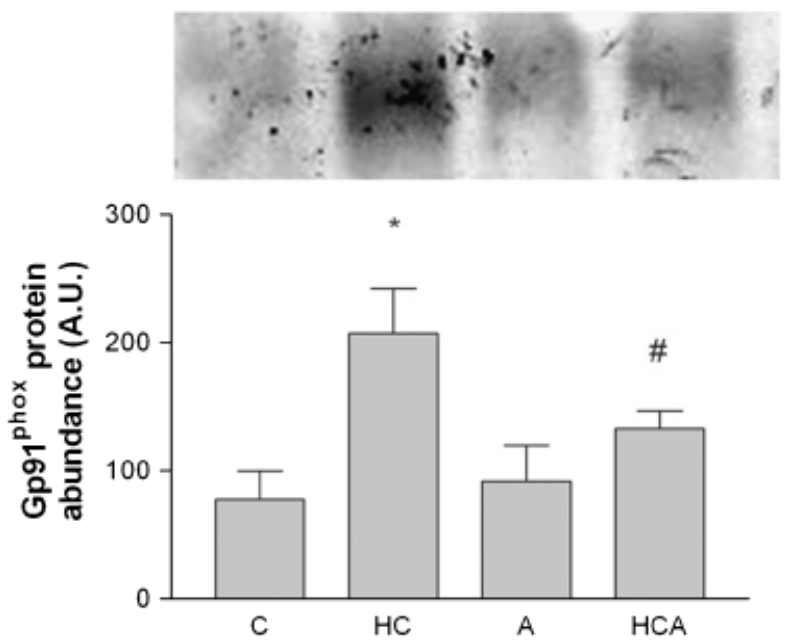

Fig. 8. Tissue expression of gp $91^{\text {phox }}{ }^{*} P<0.05$ compared with control (C); ${ }^{\#} P<0.05$ compared with high-cholesterol intake $(\mathrm{HC})$. For immunoblots, data were quantified from three independent experiments of pooled samples $(n=4$ in each group).

dinucleotide (NADP) and loses fluorescent properties. Any cellular events resulting in overproduction of oxidants would oxidize NADPH to NADP and decrease NADPH-derived autofluorescence. This method has been used to evaluate oxidative metabolism in cultured cells, and in this study we demonstrated its feasibility for in vivo liver studies. We observed decreased NADPH-derived autofluorescence in hepatocytes of hypercholesterolaemic rats. Although hypoperfusion would lower NADPH-derived autofluorescence, this was not likely the cause of the observed phenomenon owing to the well-known resistance to dietary cholesterol-induced atherosclerosis of rats and the absence of any pharmacological stress during the experiment. Inhibition of NADPH oxidase activity with apocynin resulted in an increased autofluorescence intensity, which further confirmed the relationship between cellular redox status and the autofluorescence intensity. Biochemical methods were also applied to determine the redox status of the liver. The results showed that a high-cholesterol intake increased the tissue content of TBARS and depleted glutathione in the liver, indicating an overall prooxidative state. It is interesting that $\mathrm{NADPH}$ is a cofactor for recycling GSSG to reduced one and confer endogenous anti-oxidative defense. Therefore, reduced NADPH abundance might hinder efficient glutathione cycling in hypercholesterolaemic liver.

In conclusion, the results with apocynin supported the role of NADPH oxidase in hypercholesterolaemiaassociated liver injuries. As there are high coincidences of hypercholesterolaemia and metabolic syndrome, 
hepatic cholesterol accumulation might act synergistically with excess fatty acid to accentuate the lipotoxicity of a western-style diet. Furthermore, NADPH oxidase is also implied in the pathogenesis of NAFLD in $\mathrm{fa} / \mathrm{fa}$ mice (30). Therefore, NADPH oxidase should be a potential molecular target for the development of steatohepatitis treatment strategies. In the era of affluence, NAFLD is becoming the leading cause of chronic liver diseases. Given the prevalence of hypercholesterolaemia, its role and pathogenic potential in NAFLD warrant further investigation.

\section{Acknowledgement}

This work was supported by Taiwan National Science Council grants NSC92-2314-B-002-220 to Dr C. C. Wu.

\section{References}

1. Adams LA, Lymp JF, St Sauver J, et al. The natural history of nonalcoholic fatty liver disease: a population-based cohort study. Gastroenterology 2005; 129: 113-21.

2. Marrero JA, Fontana RJ, Su GL, Conjeevaram HS, Emick DM, Lok AS. NAFLD may be a common underlying liver disease in patients with hepatocellular carcinoma in the United States. Hepatology 2002; 36: 1349-54.

3. Li D, Liu L, Chen H, Sawamura T, Mehta JL. LOX-1, an oxidized LDL endothelial receptor, induces CD40/CD40L signaling in human coronary artery endothelial cells. Arterioscler Thromb Vasc Biol 2003; 23: 816-21.

4. Li S L, Dwarakanath RS, Cai Q, Lanting L, Natarajan R. Effects of silencing leukocyte-type 12/15-lipoxygenase using short interfering RNAs. J Lipid Res 2005; 46: 220-9.

5. Li Y, Schwabe RF, DeVries-Seimon T, et al. Free cholesterolloaded macrophages are an abundant source of tumor necrosis factor-alpha and interleukin-6: model of NF-kappaB- and map kinase-dependent inflammation in advanced atherosclerosis. J Biol Chem 2005; 280: 21763-72.

6. Tabas I. Consequences of cellular cholesterol accumulation: basic concepts and physiological implications. J Clin Invest 2002; 110: 905-11.

7. Vainio S, Ikonen E. Macrophage cholesterol transport: a critical player in foam cell formation. Ann Med 2003; 35: 146-55.

8. Stokes KY, Granger DN. The microcirculation: a motor for the systemic inflammatory response and large vessel disease induced by hypercholesterolaemia? J Physiol 2005; 562: 647-53.

9. Jeong WI, Jeong DH, Do SH, et al. Mild hepatic fibrosis in cholesterol and sodium cholate diet-fed rats. J Vet Med Sci 2005; 67: 235-42.

10. Wanless IR, Belgiorno J, Huet PM. Hepatic sinusoidal fibrosis induced by cholesterol and stilbestrol in the rabbit: 1 Morphology and inhibition of fibrogenesis by dipyridamole. Hepatology 1996; 24: 855-64.
11. Cathcart MK. Regulation of superoxide anion production by NADPH oxidase in monocytes/macrophages: contributions to atherosclerosis. Arterioscler Thromb Vasc Biol 2004; 24: 23-8.

12. Carlson SE, Goldfarb S. A sensitive enzymatic method for determination of free and esterified tissue cholesterol. Clin Chim Acta 1977; 79: 575-82.

13. Kable EP, Kiemer AK. Non-invasive live-cell measurement of changes in macrophage $\mathrm{NAD}(\mathrm{P}) \mathrm{H}$ by two-photon microscopy. Immunol Lett 2005; 96: 33-8.

14. Maeda K, Yasunari K, Sato EF, Inoue M. Enhanced oxidative stress in neutrophils from hyperlipidemic guinea pig. Atherosclerosis 2005; 181: 87-92.

15. Perriott LM, Kono T, Whitesell RR, et al. Glucose uptake and metabolism by cultured human skeletal muscle cells: ratelimiting steps. Am J Physiol Endocrinol Metab 2001; 281: E72-80.

16. Rouhanizadeh M, Hwang J, Clempus RE, et al. Oxidized-1palmitoyl-2-arachidonoyl-sn-glycero-3-phosphorylcholine induces vascular endothelial superoxide production: implication of NADPH oxidase. Free Radical Biol Med 2005; 39: 1512-22.

17. Bataller R, Schwabe RF, Choi YH, et al. NADPH oxidase signal transduces angiotensin II in hepatic stellate cells and is critical in hepatic fibrosis. J Clin Invest 2003; 112: 1383-94.

18. Harada H, Hines I N, Flores S, et al. Role of NADPH oxidasederived superoxide in reduced size liver ischaemia and reperfusion injury. Arch Biochem Biophys 2004; 423: 103-8.

19. Teufelhofer O, Parzefall W, Kainzbauer E, et al. Superoxide generation from Kupffer cells contributes to hepatocarcinogenesis: studies on NADPH oxidase knockout mice. Carcinogenesis 2005; 26: 319-29.

20. Abdelrahman M, Mazzon E, Bauer M, et al. Inhibitors of NADPH oxidase reduce the organ injury in haemorrhagic shock. Shock 2005; 23: 107-14.

21. James LP, McCullough SS, Knight TR, Jaeschke H, Hinson JA. Acetaminophen toxicity in mice lacking NADPH oxidase activity: role of peroxynitrite formation and mitochondrial oxidant stress. Free Radical Res 2003; 37: 1289-97.

22. Bennett BD, Jetton TL, Ying G, Magnuson MA, Piston DW. Quantitative subcellular imaging of glucose metabolism within intact pancreatic islets. J Biol Chem 1996; 271: 3647-51.

23. Pedruzzi E, Guichard C, Ollivier V, et al. $\mathrm{NAD}(\mathrm{P}) \mathrm{H}$ oxidase Nox-4 mediates 7-ketocholesterol-induced endoplasmic reticulum stress and apoptosis in human aortic smooth muscle cells. Mol Cell Biol 2004; 24: 10703-17.

24. Feng B, Yao PM, Li Y, et al. The endoplasmic reticulum is the site of cholesterol-induced cytotoxicity in macrophages. Nat Cell Biol 2003; 5: 781-92.

25. Vilhardt F, van Deurs B. The phagocyte NADPH oxidase depends on cholesterol-enriched membrane microdomains for assembly. EMBO J 2004; 23: 739-48.

26. Mari M, Caballero F, Colell A, et al. Mitochondrial free cholesterol loading sensitizes to TNF- and Fas-mediated steatohepatitis. Cell Metab 2006; 4: 185-98. 
27. Willner EL, Tow B, Buhman KK, et al. Deficiency of acyl CoA: cholesterol acyltransferase 2 prevents atherosclerosis in apolipoprotein E-deficient mice. Proc Natl Acad Sci USA 2003; 100: 1262-7.

28. Lian X, Yan C, Yang L, Xu Y, Du H. Lysosomal acid lipase deficiency causes respiratory inflammation and destruction in the lung. Am J Physiol Lung Cell Mol Physiol 2004; 286: L801-7.
29. Tylki-Szymanska A, Rujner J, Lugowska A, Sawnor-Korszynska D, Wozniewicz B, Czarnowska E. Clinical, biochemical and histological analysis of seven patients with cholesteryl ester storage disease. Acta Paediatr Jpn 1997; 39: 643-6.

30. Carmiel-Haggai M, Cederbaum AI, Nieto N. A high-fat diet leads to the progression of non-alcoholic fatty liver disease in obese rats. FASEB J 2005; 19: 136-8. 